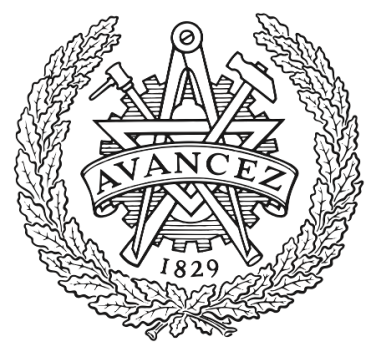

CHALMERS

UNIVERSITY OF TECHNOLOGY

\title{
Next-Generation Battery Management Systems: Dynamic Reconfiguration
}

Downloaded from: https://research.chalmers.se, 2023-04-26 07:48 UTC

Citation for the original published paper (version of record):

Han, W., Wik, T., Kersten, A. et al (2020). Next-Generation Battery Management Systems: Dynamic Reconfiguration. IEEE Industrial Electronics Magazine, 14(4): 20-31.

http://dx.doi.org/10.1109/MIE.2020.3002486

N.B. When citing this work, cite the original published paper. 


\section{Next-Generation}

\section{Battery Management}

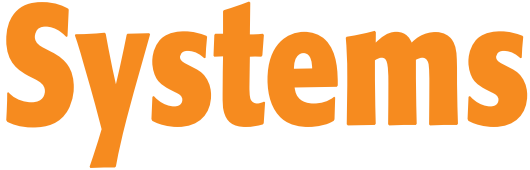

\section{Dynamic \\ Reconfiguration}

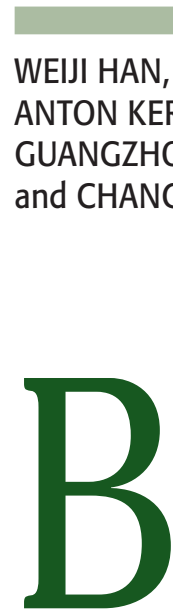

atteries are widely applied to the energy storage and power supply in portable electronics, transportation, power systems, communication networks, and so forth. They are particularly demanded in the emerging technologies of vehicle electrification and renewable energy integration for a green and sustainable society. To meet various voltage, power, and energy requirements in large-scale applications, multiple battery cells have to be connected in series and/or parallel. While battery technology has advanced significantly during the past decade, existing battery management systems (BMSs) mainly focus on the state monitoring and control of battery systems packed in fixed configurations. In fixed configurations, though, battery system performance is, in principle, limited

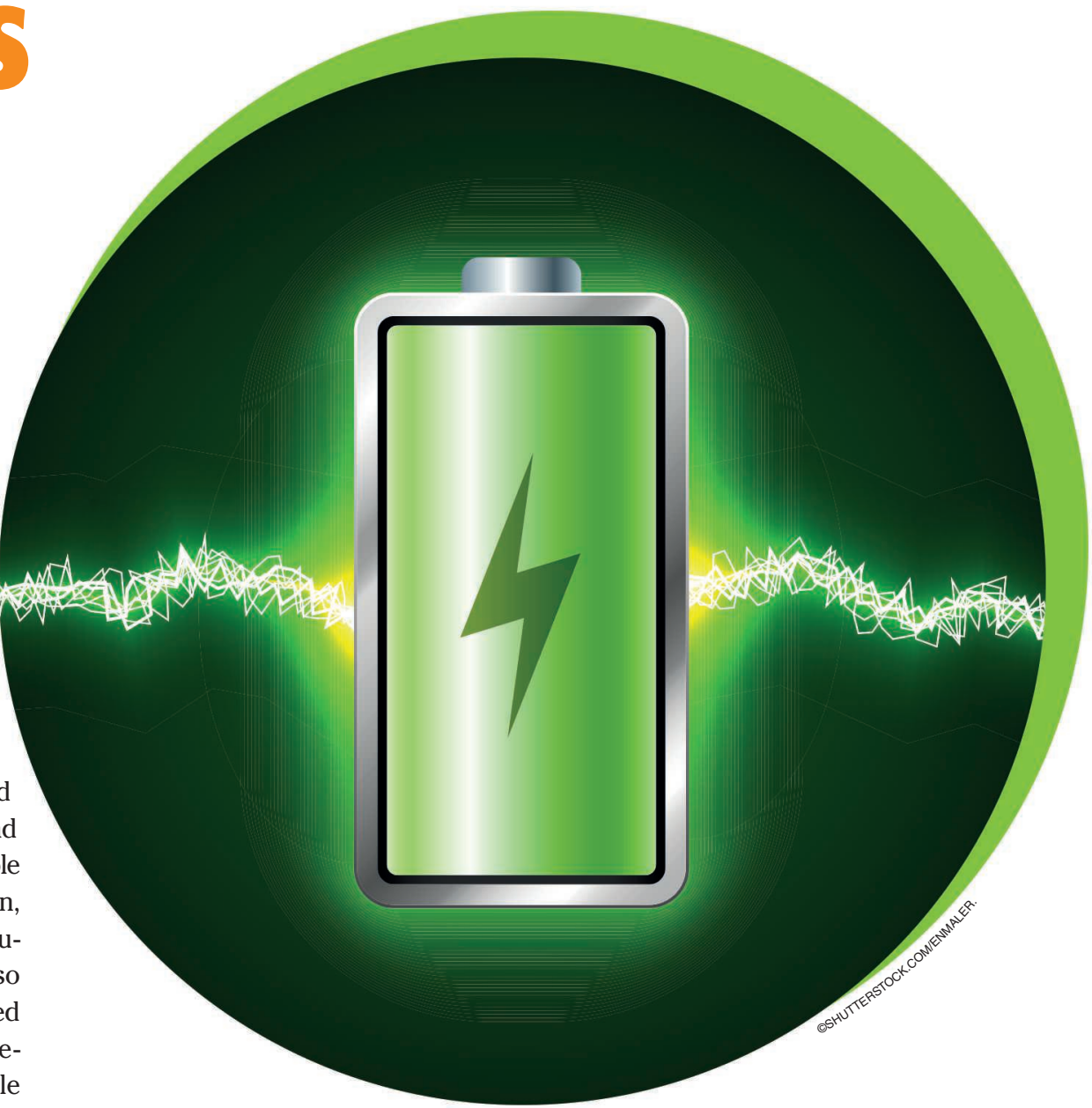

by the weakest cells, which can leave large parts severely underutilized.

Allowing the dynamic reconfiguration of battery cells, on the other hand, enables individual and flexible manipulation of the battery system at cell, module, and pack levels, which may open up a new paradigm for battery management. Following this trend, this article provides an overview of next-generation BMSs featuring dynamic reconfiguration. Motivated by numerous potential benefits of reconfigurable battery systems (RBSs), hardware designs, management principles, and optimization algorithms for RBSs are sequentially and sys- tematically discussed. Theoretical and practical challenges related to the design and implementation of RBSs are highlighted to stimulate future research and development.

\section{Functionalities and Benefits of RBSs}

RBSs, conceptually, are capable of changing the battery interconnection pattern in response to the battery behavior, state of controllable hardware components, and user demands. Figure 1 illustrates a set of RBS application scenarios. The enabled functionalities and potential benefits are summarized as follows. 


\section{Enhanced Fault Tolerance}

In a conventional battery system, the configuration is generally fixed. Cell faults, such as internal and external short circuits, may damage cells and rapidly spread to neighboring cells, gradually destroying the entire system. This will waste the energy, materials, and investment in the neighboring cells, and local cell-level faults can potentially escalate to higher levels and cause fires and explosions, leading to catastrophic consequences to battery-powered devices as well as their users. These risks can be avoided by an appropriately designed BMS equipped with dynamically controlled configurations. Specifically, RBSs are able to quickly disconnect faulty cells while reconnecting the remaining normal ones [6]-[10]. This means that local faults can be isolated in a timely manner so that other cells can keep working without significantly affecting system-level functions and performance. Figure 1(a) displays a simple battery structure designed in [11] to elucidate the fault isolation in RBSs. The malfunctioning cell $\left(\mathrm{C}_{2}\right)$ can be bypassed by manipulating the switches around it, while other cells can continue providing power to the load or absorbing energy from the charger.

\section{Charge and Temperature Balancing}

Due to inevitable variations in manufacturing techniques and different operating conditions, battery cells of the same type in a pack are inherently heterogeneous, which can be reflected by unbalanced charge and nonuniform temperature. A charge imbalance largely reduces the available capacity of series-connected multicell systems. In this circumstance, some cells will be underutilized, leading to unnecessary up-front cost, weight, and space [12], while other cells may encounter overutilization, such as overcharging and overdischarging, which is the primary reason for premature battery degradation [13] and safety issues [14]. Thermal imbalance will cause different cell aging rates and reduce the longevity of the battery system as well as potentially trigger overheating and threaten battery safety.
To address the inconsistency in battery systems that have a fixed configuration, charge balancing circuits and cooling devices have to be added to the BMS [15]-[17]. Fortunately, through system reconfiguration, the battery charge equalization process can be substantially expedited. This can be clearly seen in Figure 1(b), where, after reconfiguration, only roughly half the time is needed for cells to reach state of charge (SoC) equalization [1]. Notably, for RBSs, cell balancing is possible without any additional balancing circuitry. For instance, by changing the configuration, the cell with a larger SoC can be charged at a lower current or for a shorter period. Similarly, temperature gradients can be effectively flattened by coordinating the cell current.

\section{Extended Energy Delivery}

Motivated by the idea of battery balancing without auxiliary modules, dynamic reconfiguration can also be used to schedule the operation of batteries for faster and enhanced energy conversion during both charging and discharging. Taking the charging of a series of battery cells as an example, such a benefit can be realized by sequentially putting cells to rest once they have reached the upper voltage limit. Then, the charging rate of cells below this limit does not have to be lowered due to those cells that have already hit the cap. As a result, if the current and temperature are precisely controlled, all cells can be charged to their full capacity more rapidly. For the discharging process, appropriately scheduling the operation and rest of batteries could also improve the conversion of chemical energy into electrical power. A design of such a scheduling framework is illustrated in Figure 1(c) [2] for extended energy delivery and operation time, where the lines $c$ and $d$ represent the charging and discharging currents, respectively. By scheduling the battery operation, a substantial potential to increase the total energy delivery was also demonstrated in [18] and [19].

\section{Coordinating Batteries of Different Ages and Chemistries}

Once manufactured, batteries continuously experience aging during storage, charging, and discharging due to a number of side reactions, as reviewed in [13]. The irreversible aging process causes a decrease in the charge capacity and an increase in the internal ohmic resistance. While the former shrinks the battery energy capacity, the latter degrades the battery power capability. Because of manufacturing variations and the long-term unbalanced distribution of the $\mathrm{SoC}$ and temperature, battery cells in a pack commonly suffer from different degrees of aging, and the health of those most-aged cells will determine a system's life span. In turn, inconsistent health levels will also immediately affect the $\mathrm{SoC}$ and temperature profiles across in-pack cells. Therefore, as compared to battery charge balancing, the management problem becomes more complex for batteries of different ages. In practice, battery cells with less than $80 \%$ of their rated capacity are considered to no longer suit electric vehicle (EV) applications [20], but they may still have value as stationary energy storage, where operating conditions are gentler and the energy density requirements are less strict [3], [21]. With the intrinsic merit of balancing batteries, RBSs can not only prolong first-life usage, but they also become imperatively important for second-life applications, as shown in Figure 1(d).

A dynamically reconfigurable structure is also beneficial for managing battery cells of different chemistries. The initial idea was proposed in [4], and the technology was referred to as software-defined batteries (SDBs), as described in Figure 1(e). SDBs are supported by the fact that different commercial batteries perform better in various aspects, such as energy density, power density, life span, cost, fast charging, and energy efficiency, making them suitable for different applications. The goal for SDBs is to fully employ the strengths of different types of batteries through dynamic reconfiguration. 


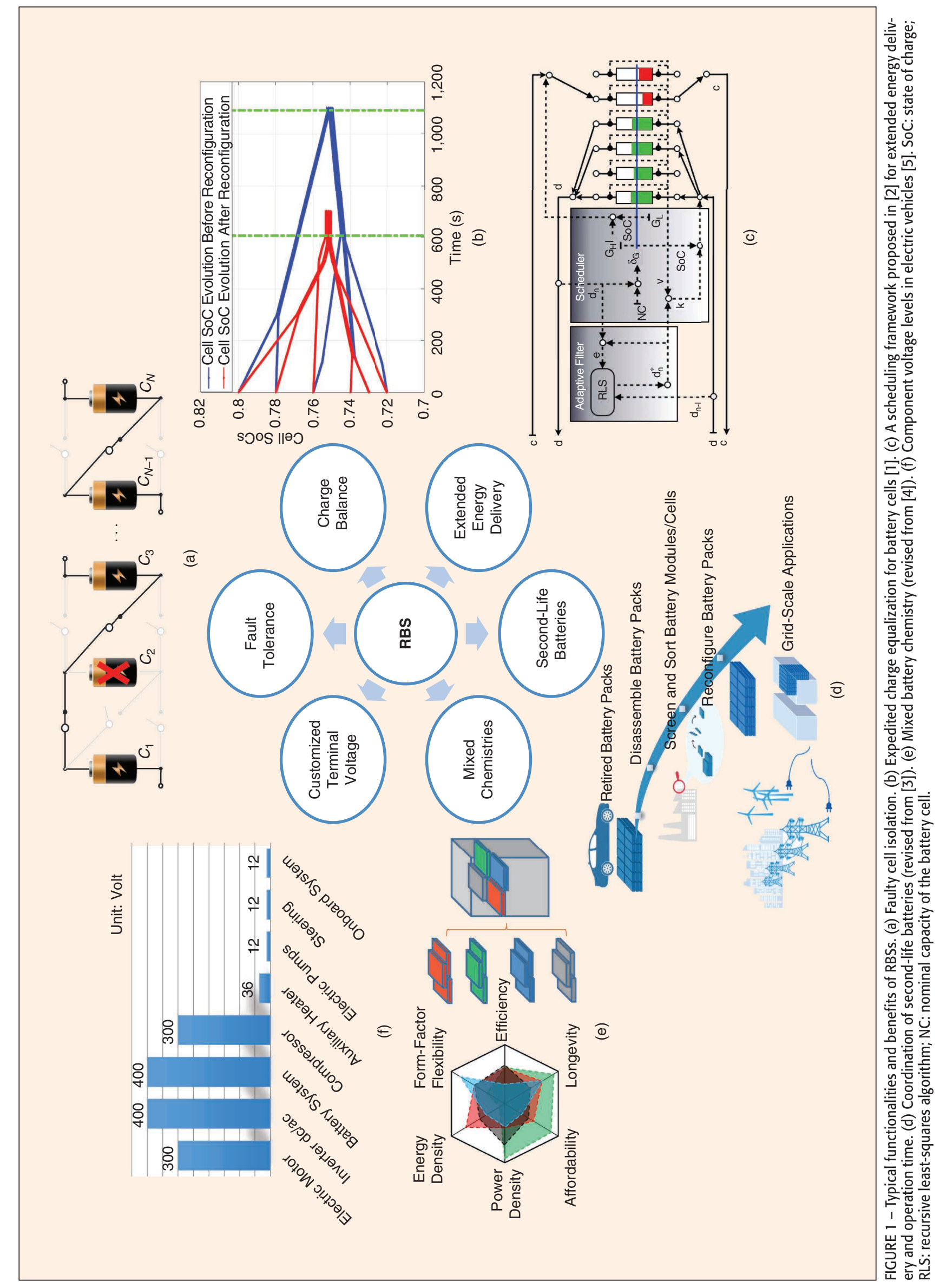

22 IEEE INDUSTRIAL ELECTRONICS MAGAZINE - DECEMBER 2020 


\section{Customized Terminal Ranges}

Figure 1(f) lists the nominal voltages for electrical components in mediumsize passenger EVs. Clearly, the voltages demanded by different components span a large range. If the traction battery system needs to provide energy for all those components, a set of inverters and converters is required, implying increased cost, weight, and system complexity, and reduced energy efficiency. An RBS is capable of customizing the terminal voltage, current, and power in across a broad spectrum. Thus, inverters and converters used for connecting the battery system to electrical components can be avoided. Likewise, the voltage range of RBS chargers can be largely extended, which, accordingly, increases the convenience for EV charging.

\section{Other Benefits}

The reconfiguration technique makes it viable to share battery modules and packs among different applications, enabling a new battery business model that is not otherwise economically justifiable and further enhancing economy and resource efficiency. This can involve cells of different SoCs, temperatures, ages, types, and chemistries, i.e., completely different cells, even within one pack. Moreover, thanks to the reconfigurability, battery cells and modules in an RBS may be separately diagnosed, repaired, and replaced, requiring much less maintenance effort and cost as compared to working on an entire battery pack that has a fixed configuration. Many battery types should not be stored at the fully discharged level, and, consequently, a battery system with a large number of cells connected in series becomes difficult and dangerous to handle due to the high voltage and energy. However, in an RBS, where cells can be flexibly disconnected, this complicating issue can be completely avoided.

\section{Summary}

The functionalities and benefits of RBSs discussed previously are all attributed to the added freedom to redistribute the battery current that results from the dynamic reconfiguration of battery cells, modules, and packs. Thus, in addition to the aforementioned advantages, all current-related performance metrics can be potentially improved and even optimized via appropriate battery system reconfiguration enabled by a next-generation BMS. This will ultimately and significantly boost system-level performance in areas such as the life span, fault tolerance, energy utilization efficiency, charging speed, power capability, and convenience of batteries.

\section{Analysis of RBS Circuit Designs}

Generally, battery system reconfiguration can be implemented in two ways, namely, relocating battery cells/ modules/packs and altering the connection/wiring. In the vast majority of commercial applications, battery cells, modules, and packs are assembled in a way that does not allow physical movement because of safety and reliability concerns. Furthermore, the average cost to change the position of batteries, in practice, can be prohibitively high. In this regard, RBS research and development efforts have mainly been devoted to changing the connection/wiring topologies.

To design an RBS, auxiliary circuit devices are imperative. Switch circuits that possess the ability to directly disconnect and reconnect individual batteries have become the most popular option. Existing switch circuits are either mechanical or semiconductor based. Mechanical switches can be implemented by relays with single contacts, changeover contacts, and multiple contacts that share one actuator [22]. The advantages are that such switches are capable of performing both forward and reverse current control. However, mechanical switches are prohibited in parallel connections since a too large current may pass one contact if some switches react faster or slower than others [23]. Unlike mechanical switches, semiconductor switches, such as MOSFETs, demonstrate good performance when connecting battery cells in parallel. Because of this, MOSFETs of low cost and high conduction efficiency have been applied to many emerging RBS designs. Comparative discussions of implementing these switch circuits can be found, for example, in the thesis [22] and the survey article [24].

The number of switches assigned to each battery cell and the switchcell connection pattern determine a system's reconfigurability. A number of RBS circuit designs in the literature [2], [6]-[11], [25]-[35] are presented in Figure 2, where two to six switches are coupled to each battery cell to realize various connection and operation possibilities. These circuit structures are further compared in Table 1, in terms of the number of switches per cell, achievable connections and operations, and reported advantages over traditional battery systems. For notational convenience, $\mathrm{S}(\mathrm{P})$ is used

\begin{tabular}{|c|c|c|c|c|}
\hline $\begin{array}{l}\text { DESIGN } \\
\text { DIAGRAM }\end{array}$ & REFERENCE & $\begin{array}{l}\text { SWITCHES } \\
\text { PER CELL }\end{array}$ & $\begin{array}{l}\text { CONNECTIONS } \\
\text { AND OPERATIONS }\end{array}$ & $\begin{array}{l}\text { REPORTED } \\
\text { BENEFITS }\end{array}$ \\
\hline Figure 2(a) & {$[26],[27]$} & Two & $\underline{S}$ & 1), 2), 3) \\
\hline Figure 2(b) & [25] & Two & $\underline{S}$ & 2), 3), 4) \\
\hline Figure 2(c) & [10] & Three & $\underline{S}$ & 1), 3), 4), 6) \\
\hline Figure 2(d) & [28], [29] & Two & $\underline{S}$ & 1), 3) \\
\hline Figure 2(e) & {$[9],[30]$} & One or two & $\underline{\text { PS }}$ & 1), 2), 3) \\
\hline Figure 2(f) & [11] & Three & $\underline{S}, P, P \underline{S}$ & 2), 4) \\
\hline Figure 2(g) & {$[36],[37]$} & Three & $\underline{S}, \underline{P}, \underline{P} 1 \underline{S}, \underline{S}, \underline{P}$ & 1), 3), 5), 6) \\
\hline Figure 2(h) & [31], [32] & Three & $S, \underline{P}, S \underline{P}$ & 2), 4) \\
\hline Figure 2(i) & {$[6],[33],[38],[39]$} & Four & $\underline{\mathrm{S}} \underline{\mathrm{P}} \underline{\mathrm{P}} \underline{\mathrm{S}}, \underline{\mathrm{SP}}$ & 2), 4), 6) \\
\hline Figure 2(j) & [7], [34] & Five & $\underline{\mathrm{S}}, \underline{\mathrm{P}} \underline{\mathrm{PS}}, \underline{\mathrm{SP}}$ & 1), 4) \\
\hline Figure 2(k) & {$[2],[8],[35]$} & Six & $\underline{\mathrm{S}} \underline{\mathrm{PS}}, \underline{\mathrm{SP}}$ & 1), 3), 4), 6) \\
\hline
\end{tabular}


to indicate the series (parallel) connection of battery cells. SP (PS) indicates that cells can be connected in series (parallel) to form a module and that an obtained module can be connected in parallel (series). The underscoring of $\mathrm{S}(\mathrm{P})$ indicates that any cell/module connected in series (parallel) can be individually bypassed. A hat under $\mathrm{S}(\mathrm{P})$ indicates that cells/modules connected in series (parallel) can be bypassed but in unison with others. A subscript "1" indicates that only one module can have multiple cells connected in series (parallel). Based on the circuit designs in Figure 2, the following benefits have been attributed to these RBSs relative to their fixed-configuration counterparts: 1) enhanced fault tolerance and safety, 2) improved energy efficiency, 3) more balanced system operation, 4) increased charge delivery/storage and a longer operating time, 5) a prolonged battery life span, and 6) customized terminal voltages.

Several observations can be made from Figure 2 and Table 1. The designs in Figure 2(a)-(d) are focused on connecting all battery cells in series, and any cell is allowed to be bypassed/ isolated by associated switch operations. By expanding each cell in Figure 2(a) to a module, the architecture in Figure 2(e) is obtained, in which a parallel cell connection is attainable but still restricted within a local module. In Figure 2(f)-(k), with at least three switches per cell, all battery cells can be connected in series, parallel, and more complex ways. For example, the cells can be connected in series (parallel), at first, to form modules, and the modules can then be joined in parallel (series). To test various possible connections and operations, as well as to develop reconfiguration strategies, a prototype of the RBS structure in Figure 2(f) was assembled using H-bridges in our battery lab, as illustrated in Figure 3. When at least five switches are assigned to each battery cell, e.g., via the designs in Figure 2(j) and (k), battery cells can be flexibly reconfigured among the $\mathrm{S}$, P, SP, and PS connections, and any cell can be bypassed, if requested.

It is worth noting that, even using the same number of switches per cell [e.g., three switches per cell applies to the architectures in Figure 2(c), (f), (g), and (h)], we can achieve very different connection topologies, depending on how these switches are connected to the cell. Thus, both the number of switches per cell and the type of switch-cell connection contribute to the system reconfigurability. In addition, the system complexity and overall cost of RBS circuit designs depend heavily on the number of switches. To design scalable RBSs and retain the freedom for cell control, an identical number of functional switches and a uniform connection pattern should be applied to all battery cells (except those located at the boundaries). This will effectively reduce the complexity of the system design, mathematical modeling, and control algorithms for RBS management.

By carefully comparing and analyzing various reconfiguration designs, it can be found that the switches around each battery cell can be divided into different groups to realize specific connections, such as series connections, local parallel connections with neighboring cells/modules, global parallel connections of all cells/modules, and bypass connections. These switch groups can operate independently, exclusively, and jointly. Grouping switches based on achievable connections will shed new light on the design of RBSs. Then, per the need for system reconfigurability, one can actively select corresponding groups of switches and estimate the necessary number of switches for cost assessment.

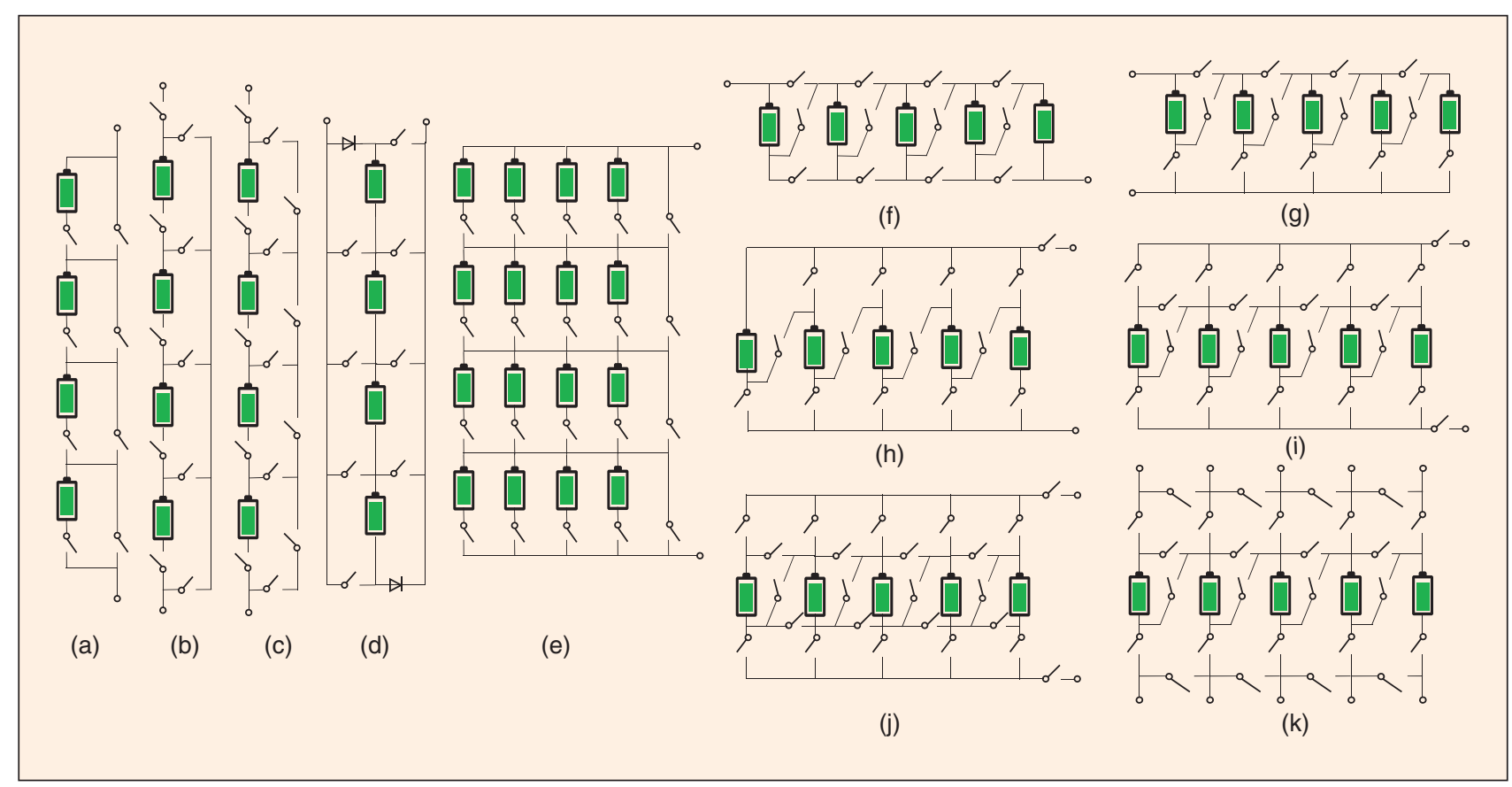

FIGURE 2 - Various RBS circuit designs proposed in recent literature (see Table 1). 


\section{Management Principles of Battery System Reconfiguration}

The various RBS circuit designs analyzed in the preceding pave the way for pursuing a number of potential benefits, some of which have been reported in Table 1. Once an RBS circuit design is complete, appropriate management strategies need to be developed to identify and realize the configurations that yield the desired system performance. To do so, management principles for battery reconfiguration in different scenarios are discussed in this section.

\section{Principle for Fault Isolation}

For the continuous and safe operation of RBSs, it is crucial to isolate any faulty, overcharged, and overdischarged battery cells without interrupting others. This functionality has been widely applied in RBSs [6]-[10], [26], [28], [30], [40]. While battery isolation and skipping can be realized in most of the RBS designs in Figure 2 (indicated by the underlined connections in Table 1), different switch operations may be performed, depending on the circuit design. When battery cells are connected in parallel, e.g., the design in Figure 2(e), it is easy to skip a cell by operating only one switch. If the cell to be isolated lies in a series-connected string, e.g., in Figure 2(a)-(c), at least two switch operations must be simultaneously conducted. To isolate a cell in RBSs that have more switches per cell and mixed series/parallel connections, e.g., in Figure 2(f)-(k), sufficient care should be taken to coordinate multiple switch operations so that no open and short circuits are incurred.

\section{Scheduling Principle for Charge Balance}

In addition to passively skipping faulty battery cells, RBSs can tackle the charge imbalance issue by actively scheduling the operation tasks of normal batteries. Aimed at more balanced operation, the basic principle of such battery scheduling is to periodically prioritize the charging (discharging) of battery cells with lower (higher) charge. To do so, the first step is to sort all battery cells according to their amount of charge, which, unfortunately, cannot be physically measured. Thus, different alternatives are applied to battery sorting, e.g., the cell's SoC [2], [9], [18], [27], [41], [42], open circuit voltage (OCV) [25], and terminal voltage [19], of which the former two are usually used in combination with state estimation.

After sorting, in response to the total charging/discharging demand, battery cells are selectively put into use following the preceding principle for balanced operation. Note that battery sorting should be periodically or adaptively updated according to battery state dynamics. As a result, the charge levels of battery cells can gradually be balanced, and, meanwhile, performance improvements arising from the charge balance can be achieved.

\section{Scheduling Principle for Enhanced Energy Conversion}

Appropriate battery scheduling can also help deliver more energy or charge during discharging, corresponding to a higher conversion efficiency from a battery's chemical energy to electrical energy. To accomplish this, the reconfiguration principle can be derived based on the recovery effect; i.e., a battery's terminal voltage during discharging can quickly recover if the battery is allowed to rest for a while or discharge at a lower current rate [2], [10], [18]. Voltage recovery is mainly determined by the battery SoC, discharging rate, and scheduled operating and resting time. To take advantage of this effect, a simple scheduling principle was developed in [18] for discharging a battery system consisting of four packs. The general principle is to periodically detect and rest the battery pack with the lowest charge while continuing to discharge the others. Following this, the total energy delivery, from fully charged to completely discharged, can be extended,

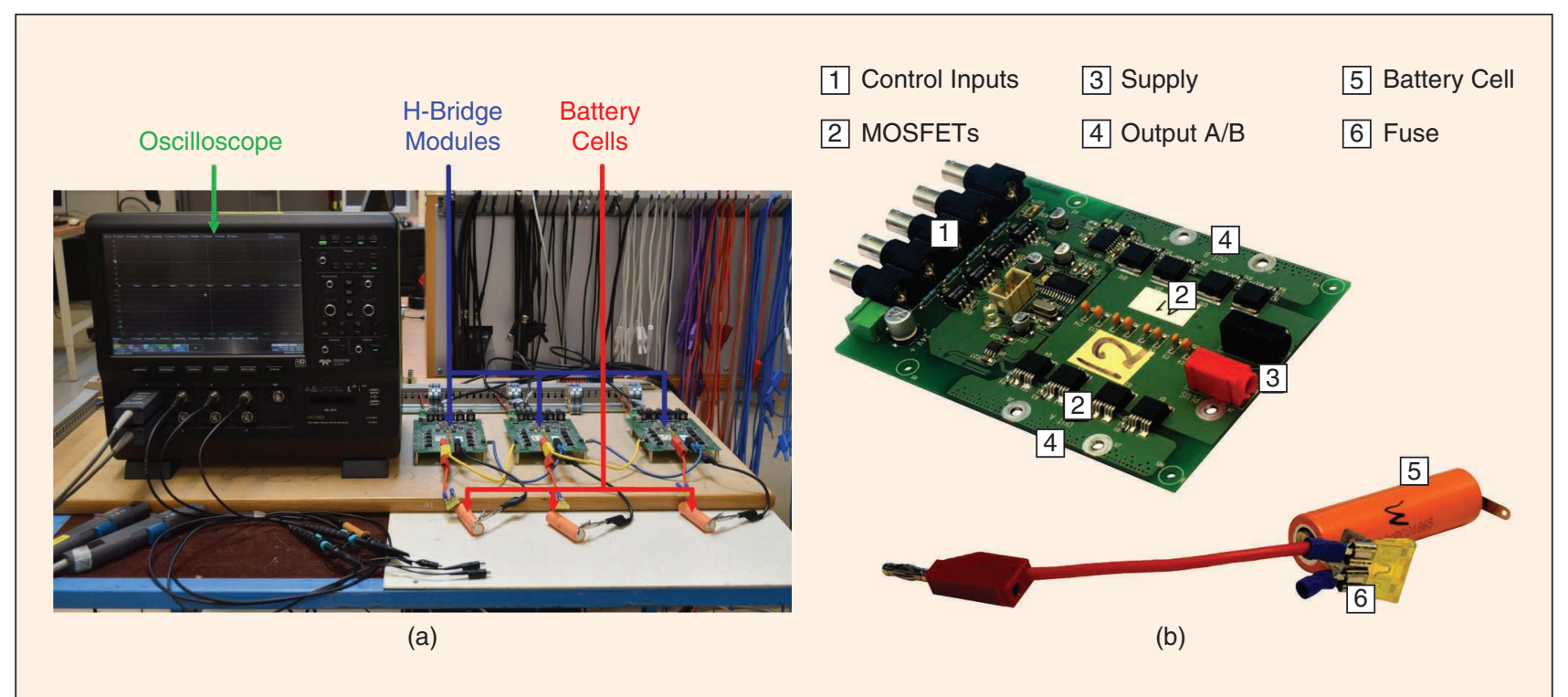

FIGURE 3 - A prototype made of three battery cells connected through three H-bridges, according to the RBS design in Figure 2(f). (a) The general setup. (b) The H-bridge and the battery cell. 
as compared to the case when all packs are always discharged without any scheduled rest. For a quantitative investigation, the extended energy deliveries under different constant load power levels and resting periods are identified and compared in Figure 4, based on experimental test data gathered from [18].

It can be seen from Figure 4 that the total energy delivery can be increased in all these tests due to scheduled resting. Given the same resting period, the extension demonstrates a decreasing trend in response to an increased load power, e.g., the four orange cones with a resting period of 15 min. The load power level's influence derives from the battery ratecapacity effect; i.e., the higher the discharging rate, the lower the charge/ energy delivery [2], [10], [38], [43], [44]. Thus, when choosing the group of batteries to be scheduled to rest, a tradeoff has to be made between the resting batteries' recovery effect and the operating batteries' rate-capacity effect. This was discussed in [27] and quantitatively examined in [2].

In addition, as documented in Figure 4 , the scheduled resting period has significant influence on the extended energy delivery. The majority of battery voltage recovery can be achieved very quickly, e.g., within 1 min for battery packs in [18], and, consequently, additional resting time for the lowest-charge pack does not contribute much to the recovery. However, a longer resting period corresponds to a longer operating period and larger voltage drops for those operating packs, which makes it easier for the packs to hit the lower voltage limit. Therefore, when designing the scheduling principle for extended energy delivery, appropriate resting and operating periods should be carefully selected based on the requested load power level. The total energy delivery for a wide range of resting periods was also experimentally tested and compared in [19], indicating that it is possible to maximize the total energy delivery at certain resting periods.

\section{Principle for Improving Circuit Energy Efficiency}

The energy efficiency of an RBS depends not only on the battery energy conversion discussed previously but also the energy loss of all involved circuit components. The switch circuit plays an essential role in battery system reconfiguration. Its power loss includes losses in the MOSFET switches and gate drive circuits. The former are composed of conduction and switching losses, and the latter depend on the specific gate drive circuit design. For instance, for the switch circuit design in [9], as the discharging current increases, the total power loss of the switch circuits decreases, at first, and then increases. Thus, there exists a discharging current leading to the maximum power efficiency of the switch circuits.

In addition, the RBS enables a flexible and wide-ranging system terminal voltage, and the load/charger voltage can also change through time. To

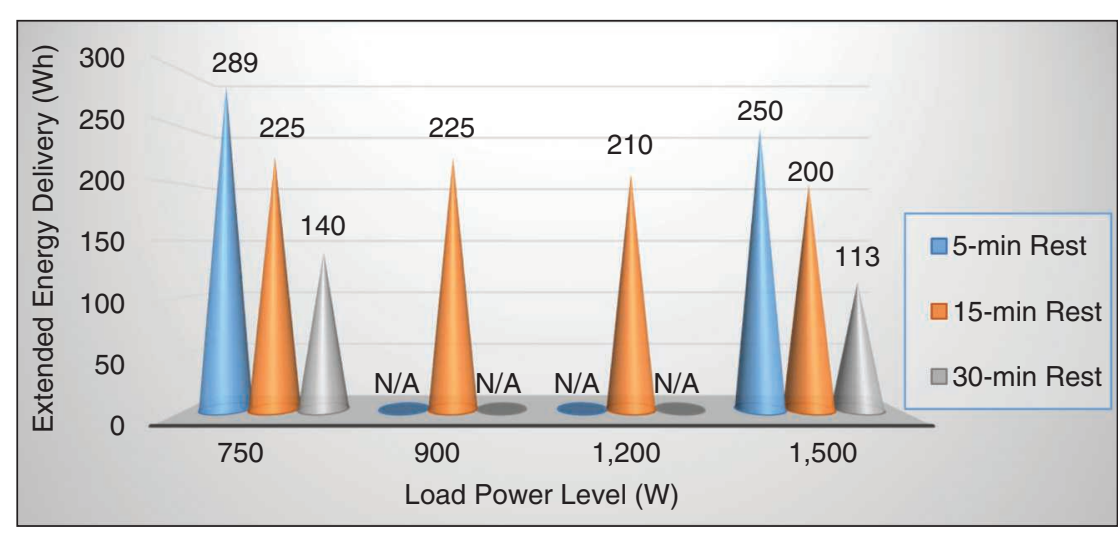

FIGURE 4 - A comparison of extended energy delivery at various load power levels and scheduled resting periods for a battery system composed of four reconfigurable packs. Note that zero resting time corresponds to zero extended energy delivery, by definition. buffer the voltage mismatch between the RBS and the load/charger, voltage regulators or power converters are needed, which introduces additional power conversion losses [11], [31], [33], [43]. Such voltage regulator losses can vary dramatically. As pointed out in [31], the conversion efficiency can drop below $50 \%$ in light load conditions. The regulator's efficiency depends on its input voltage, i.e., the RBS terminal voltage [11], [31]. Thus, in pursuit of the higher efficiency of these circuit components, the common practice is to first evaluate the efficiencies of these components at various voltages and currents and then comprehensively select the configuration that places the operation in the high-efficiency operating range while meeting the charging/discharging requirement in real time.

\section{Additional Principles}

Other principles of battery system reconfiguration can also be developed. For example, principles for faster charge equalization through analyzing performance evaluation formulas can be found in [1] and [45], and principles for distributed control based on consensus protocols have been presented in [29] and [46]. These heuristic RBS management principles are aimed to achieve more robust, balanced, and efficient system operation. However, the optimal performance of RBSs may not be attained unless the RBSs are equipped with advanced optimization and control algorithms, as detailed in the following section.

\section{RBS Modeling and Optimization Algorithms}

To maximize the benefits of RBSs while ensuring safety and reliability, optimization algorithms need to be developed. To do so, the fundamental step is to devise a mathematical model for RBSs.

\section{Modeling RBSs}

In an RBS, battery cells, switches, and their interconnection topology all significantly influence the system performance. Therefore, it is necessary to establish mathematical models 
capable of accurately predicting the evolution of critical system states.

There are three general classes of battery cell models, i.e., physics based, equivalent circuit based, and data driven. As mentioned, a vast number of battery cells can be involved in large-scale RBS applications. How to mathematically model such complex systems, from the cell level to the module and pack level, with a good balance between computational efficiency and accuracy is a key problem. Physicsbased models have been widely studied in academia to describe the internal dynamics of a lithium-ion battery cell, including ion diffusion and intercalation/deintercalation processes. Based on the porous electrode theory and concentrated solution theory, an initial model was proposed in [47], consisting of a set of partial differential-algebraic equations (PDAEs). The PDAE-based battery model was reformulated and simplified, e.g., in [48] and [49], to facilitate cell-level simulation and control applications. However, to apply physics-based models to BMSs, further efforts are needed to reduce the complexity of the computation and parameterization.

With the advent of the big data era, data-driven battery models have recently become popular. The main research focuses of such models have been on data-based prediction and estimation of battery's SoC [50], state of health [51], and remaining useful life [52]. Equivalent circuit-based models (ECMs), on the other hand, are relatively simple to parameterize and implement in battery pack control [53] and hence are preferred in today's BMS designs. The primary drawback of ECMs concerns a lack of physical insights directly relating to battery safety and health, such as the local overpotential and the solid electrolyte interphase film.

To model a battery system with multiple cells connected in a fixed configuration, a simple and widely applied industrial practice is to view the pack as one virtual cell. Specifically, the cell-level model is still used, but the model parameters are identified based on the pack behavior [54]. In an RBS, since both series and parallel battery connections can be flexibly reconfigured, each battery cell has to be individually modeled to adapt to different system configurations. At the same time, modeling each cell will facilitate characterizing the cell-level imbalances in terms of charge [55], impedance, and capacity; analyzing the cell current distribution under various system configurations; and designing proper RBS optimization and control algorithms. These benefits are, however, achieved at the cost of dramatically increased model complexity.

Switches are essential components to enable the reconfigurability of battery systems and can be realized through different circuit elements. In switch modeling, the connection changes are, of course, most important, but the electrical and thermal characteristics during conduction, in general, are also significant. MOSFET switches outperform mechanical ones in terms of a much lower power demand for actuation and better synchronization for parallel connections [22]. For MOSFET switches, one important parameter is the drain source on-resistance, normally denoted by $R_{D S \text { (on) }}$ and defined by the total resistance in the conductive path from the source to the drain when the MOSFET is turned on. The $R_{D S(\text { on) }}$ consists of a series of subresistances and demonstrates a high dependence on the real-time junction temperature, drain current, and gate-to-source voltage [56]-[58]. As a result, it becomes challenging to model the exact dynamic behavior of switches. In some studies, for simplicity, $R_{D S \text { (on) }}$ is ignored [25] or assumed to be constant [37]. However, accurate switch modeling is still worth exploiting when $R_{D S(\text { on) }}$ is comparable with the internal resistance of battery cells and imposes considerable influence on the total resistances.
The remaining task is to model various interconnections of battery cells through switches. A natural way to describe the interconnection of battery cells and switches is by a graph representation, state vector, or state matrix. For instance, consider the small RBS in Figure 5: the system configuration, composed of only cell 1 and cell 3 , can be represented by the edge information in a graph, i.e., $\mathcal{E}=\left\{n_{+} \rightarrow c_{1}, c_{1} \rightarrow c_{3}, c_{3} \rightarrow n_{-}\right\} ;$a cell state vector $\left(c_{1}, c_{2}, c_{3}\right)=(1,0,1)$; or a switch state vector $\left(s_{1}, s_{2}, \ldots, s_{8}\right)=$ $(1,0,1,1,0,1,1,0)$, where zero and one indicate the disconnected and connected states, respectively.

Finally, an entire RBS can be modeled by wrapping up all component models. Given any specified system configuration of an RBS, the open circuit elements are deactivated, and the electrical interaction among the remaining active battery cells and switches is characterized following Kirchhoff's current law and voltage law. Such an RBS model will act as the basis for model-based performance optimization in the subsequent section.

\section{Optimization Algorithms \\ Based on Reconfiguration}

To date, only a few studies have been conducted to improve RBS performance through model-based optimization algorithms. The common goal of these studies is to identify the system configuration that yields the best performance. Table 2 summarizes the battery reconfiguration optimization problems in recent literature. In these problems, the total charge/energy delivery (storage) during discharging (charging) or the power/energy loss has been regarded as the objective function. Other benefits arising from battery system reconfiguration, such as speeding up the charge balancing

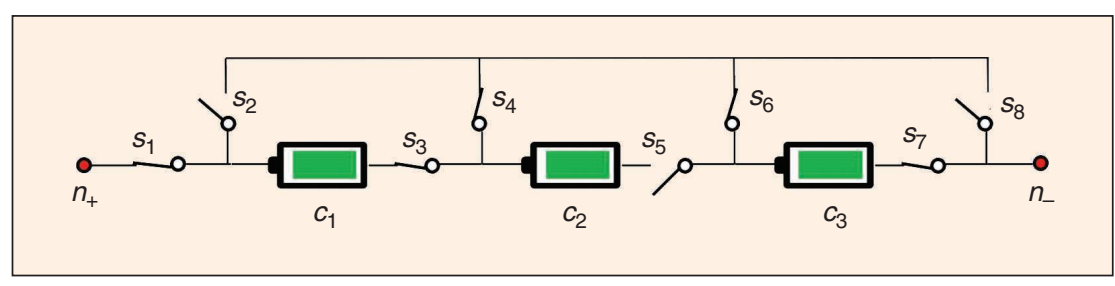

FIGURE 5 - A three-cell RBS based on the design in Figure 2(b). 
[1], [45], are also possible to quantify and maximize. These objective functions are often subject to three types of constraints to ensure safe and efficient operation of RBSs, as summarized in the following:

- electrical and thermal constraints enforced on batteries and switches to guarantee their operation within appropriate ranges of current, voltage, power, and temperature; most constraints in the referred works in Table 2 fall into this type.

- restraints on the system configuration, e.g., assigning the same number of cells to each string [11]

- Requirements of sequential or synchronized switch operations to avoid any circuit faults.

Among the problems in Table 2, those formulated in [11], [25], [33], and [43] focus on only one period, either the present control period or the full discharging process. To solve such optimization problems, graph theory has been deployed based on the graph representation of system configurations. For example, minimizing the total additional resistance was transformed to a minimum path cover problem in [25], and maximizing a battery pack's charge delivery was achieved by constructing and solving a maximum-weight independent set problem
[43]. In [11], power loss curves and terminal voltages of different system configurations were evaluated offline in advance and subsequently used for determining the optimal configuration online in response to voltage and current demands.

When it is desired to optimize the overall system performance across successive control periods, as in [34], [38], [42], [44], and [59], the system evolution has to be considered, including the time-varying OCVs, SoCs, and total available charge or energy of the cells. To take this into account, a dynamic system model needs to be appended to the optimization problem as a constraint. Such an optimization problem can be regarded as a typical optimal control problem, where some performance goals can be accomplished by applying variable constraints. For instance, to eventually achieve charge balance, the charge difference among battery cells is forced to zero at the end of the last control period [42].

Basically, the formulated optimal control problems can be solved either numerically, for example, using Bellman's dynamic programming (DP), or analytically, using Pontryagin's maximum principle [60]. The problems proposed in [34], [42], [44], and [59] are all solved by DP, a common tool

\begin{tabular}{|c|c|c|c|}
\hline REFERENCE & OBJECTIVE FUNCTION & CONSTRAINT & $\begin{array}{l}\text { METHOD OR } \\
\text { ALGORITHM }\end{array}$ \\
\hline [25] & $\begin{array}{l}\text { Minimize the total } \\
\text { additional resistance }\end{array}$ & $\begin{array}{l}\text { Charging current and number } \\
\text { of cells per string }\end{array}$ & $\begin{array}{l}\text { Graph theory-based } \\
\text { method }\end{array}$ \\
\hline [11] & $\begin{array}{l}\text { Minimize the total power } \\
\text { loss during one control } \\
\text { period }\end{array}$ & $\begin{array}{l}\text { System configuration, switch } \\
\text { operation, and battery bank's } \\
\text { voltage and current }\end{array}$ & $\begin{array}{l}\text { Looking up tables } \\
\text { prepared offline }\end{array}$ \\
\hline [33] & $\begin{array}{l}\text { Minimize the discharging } \\
\text { current of individual cells }\end{array}$ & Terminal voltage range & $\begin{array}{l}\text { Graph theory-based } \\
\text { method }\end{array}$ \\
\hline [43] & $\begin{array}{l}\text { Maximize the deliverable } \\
\text { charge capacity }\end{array}$ & $\begin{array}{l}\text { Each cell belongs to one and } \\
\text { only one string. }\end{array}$ & $\begin{array}{l}\text { Graph theory-based } \\
\text { method }\end{array}$ \\
\hline [34] & $\begin{array}{l}\text { Minimize the total charge } \\
\text { consumption }\end{array}$ & Terminal current and voltage & $\begin{array}{l}\text { Lagrangian relaxation and } \\
\text { dynamic programming } \\
\text { (DP) }\end{array}$ \\
\hline [38] & $\begin{array}{l}\text { Maximize the charge } \\
\text { delivery during discharging }\end{array}$ & Total load power & DP and genetic algorithm \\
\hline [42] & $\begin{array}{l}\text { Minimize the total energy } \\
\text { loss during charge balancing }\end{array}$ & $\begin{array}{l}\text { Total energy delivery and } \\
\text { number of cells applied }\end{array}$ & DP \\
\hline [44], [59] & $\begin{array}{l}\text { Minimize the total capacity } \\
\text { loss }\end{array}$ & $\begin{array}{l}\text { Battery cell states, load } \\
\text { profile, and working } \\
\text { conditions }\end{array}$ & $\begin{array}{l}\text { Lagrangian relaxation } \\
\text { and DP }\end{array}$ \\
\hline
\end{tabular}

for solving multistage optimization problems. When solving these optimal control problems formulated for RBSs, the computational time and memory requirements are influenced by a number of factors, such as the system dimension, model nonlinearity, number of feasible configurations, and control horizon. Consequently, online applications of these optimal control methods easily become infeasible for large-scale RBSs and/or long control horizons. To reduce the computation burden, an alternative optimization problem with respect to load power classes, instead of successive control periods, was constructed in [42], at the cost of sacrificing the global optimality of the original problem. In general, real-time applications of optimal RBS control are at a preliminary stage. There exist some challenges in formulating appropriate RBS optimization problems with detailed expressions of objective functions and constraints and developing computationally efficient algorithms to solve them.

\section{Challenges and Outlook}

While system reconfiguration promises to bring various benefits toward advanced battery management, several critical challenges need to be addressed during the hardware design, algorithm development, and switch operations.

\section{Hardware Design}

In hardware design, both the system structure and the corresponding components need to be determined. During this process, a number of design factors have to be comprehensively considered, including the requested functionalities, specific constraints on the entire RBS, and rating of all electrical components involved. When designing battery systems that have a fixed configuration, the series and/ or parallel connection structure of battery cells can be quickly determined according to constraints on the entire system, e.g., the ranges of the terminal voltage and current, required power capability, energy capacity, and life span, as well as space and weight limits. However, to seek the desired 
functionalities and benefits of RBSs, e.g., charge balancing and enhanced energy delivery, a new task is to identify the RBS structure candidates supporting them. This task initiates the design of RBSs but has never been systematically studied. Some clues can be found in Table 1, based on the association of various design structures with their enabled connections and operations. Further research efforts are expected to guide the structure selection for achieving various potential benefits of RBSs.

Another task is to choose appropriate components, such as batteries, switches, sensors, and cables, so as to fit into the candidate RBS structures. Well-designed sensing and fault tolerance mechanisms along with associated hardware resources also need to be in place to tackle faulty parts and protect the remaining components. Since a large number of interconvertible configurations can be enabled in an RBS, the rating of all electrical components and the fault tolerance design have to be performed for all feasible configurations within various working scenarios. This can become very time consuming for large-scale systems and leaves a challenging research gap in RBS design. Future investigation should be aimed at first identifying an appropriate set of desired and feasible configurations by prohibiting those potentially unsafe and unnecessary designs. Then, the rating of various components can be focused on the selected configurations in corresponding working conditions.

Furthermore, the rating of components can be extended to cover an RBS's high-efficiency operating conditions, and high-quality or redundant components can be deployed to improve reliability. These, however, normally contribute to an increased cost, which is an important concern in RBS design. Thus, a tradeoff has to be made between system efficiency, reliability, and expense. Although such a compromise is also necessary for designing battery systems of a fixed configuration, it becomes more complicated in designing RBSs, due to the presence of many more operating scenarios and components. A preliminary attempt has been made for battery pack sizing in [61], where the overall cost of cells, sensors, and controllers was analyzed and reduced while providing the required power and reliability. Following this thread, it is important to develop a generic framework in the future to strike a balance among all design factors of concern.

\section{Development of Optimization Algorithms}

After setting up a selected RBS hardware design, the performance can be optimized by manipulating the system's configuration. To develop such optimization algorithms, the system configuration is viewed as the decision variable; i.e., the cell interconnection is not deterministic. Consequently, circuit laws are difficult to apply to model the system operation, and the objective function generally lacks an explicit dependence on the system configuration. These two aspects make it challenging to formulate the optimization problem as a detailed analytical expression.

Due to the preceding features and the imposed state constraints, gradient-based methods cannot be directly applied, and thus numerical methods have to be deployed. Given any possible and feasible system configuration, a battery system model can be accordingly constructed, and the resulting system performance can be evaluated by simulation and analytical expressions [1], [45]. Then, the best performance along with the corresponding configuration can be identified through an exhaustive search if the system dimension is relatively small or by heuristic algorithms, such as genetic algorithms. Such performance evaluation and searching can become very time consuming for large-scale systems that have a huge number of possible configurations, and a globally optimal solution cannot be guaranteed. For instance, to evaluate the minimum charge equalization time of a reconfigurable battery series with 15 cells by exhaustive searching, the average computational time is estimated to be more than two years
[45]. Therefore, when applying these numerical search methods to RBS performance optimization, substantial attention should be paid to computational efficiency, especially for online applications within a BMS.

\section{Switch Operations}

Once the desired system configuration is determined for an established RBS, the original configuration will be converted to the desired one through a set of sequential and/or synchronized switch operations. These switch operations should be well coordinated for efficient implementation but without causing any short circuits and unintended open circuits. Ideally, all involved switch operations can be simultaneously performed to directly set up the desired configuration. For example, to isolate cell $c_{2}$ in Figure 5 without interrupting other cells, both connecting switch $S_{5}$ and disconnecting switches $S_{4}$ and $S_{6}$ should be concurrently executed. In a practical reconfiguration, however, switch operations may not be well synchronized, due to control signal delays and/or hardware limitations. This can cause undesired transient system behaviors, e.g., a high transient current endangering related components. Such practical issues must be carefully addressed by accurately modeling the dynamic behaviors of all interconnected components. However, this is still a challenging task because of the limited modeling fidelity of components and substantial simulation time.

Moreover, when performing sequential switch operations in RBSs, a certain time delay is required between every two successive operations. The more sequential switch operations there are, the longer the reconfiguration time will be. As a consequence, some urgent actions, such as isolating a faulty cell, might be delayed, resulting in serious safety issues and hardware damage. For such systems, heuristic suggestions are given in [61] to reduce the reconfiguration time, such as limiting the reconfiguration within a small area and reducing the number of battery packs to be reconfigured. Additionally, as demonstrated in [18], [19], 
and [61], the reconfiguration frequency also influences the RBS performance, e.g., the total energy delivery, and the optimal frequency is possible to identify through a large number of experimental tests [19] and simulations based on high-fidelity models. Despite these preliminary explorations, comprehensive guidelines for safe and efficient reconfiguration in complex and large-scale RBSs are absent.

\section{Conclusion}

Compared to fixed configurations, dynamic reconfiguration of battery systems at the pack, module, and cell levels have great potential to improve system performance from many aspects, e.g., fault tolerance, energy utilization, fast charging, and life spans. Based on this, BMSs that have the freedom of dynamic reconfiguration open up a new path to enhanced energy storage and conversion in various applications, including EVs and power grids. This article provided a critical and comprehensive overview of RBSs. After analyzing a variety of circuit designs for RBSs, we devoted the majority of our efforts to the principles for managing RBSs and the algorithms for optimizing the performance. Several critical challenges in RBS hardware design, algorithm development, and switch operations were identified and discussed. To address them, future research and development directions were highlighted. In view of the fairly scarce resources in the current literature for systematical and comprehensive studies of RBSs, this article was intended to inspire innovative thinking from both researchers and engineers on present designs and to motivate more advanced reconfiguration technology for next-generation BMSs.

\section{Acknowledgments}

This work was supported, in part, by Mistra Innovation, under project MI2319.03, and by Chalmers Transport Area of Advance.

\section{Biographies}

Weiji Han (weiji.han@chalmers.se) received his $\mathrm{Ph} . \mathrm{D}$. degree in electrical engineering at the University of Con- necticut, Storrs, USA, in 2018. He is a researcher in the Department of Electrical Engineering, Chalmers University of Technology, Gothenburg, Sweden. His research interests include the modeling, analysis, and control of battery systems with applications to electric vehicles and power grids. He has additional research experience with power, photovoltaic, and manufacturing systems.

Torsten Wik (torsten.wik@chalmers .se) received his Ph.D. degree in automatic control from Chalmers University of Technology, Gothenburg, Sweden, in 1999. He is a professor and the head of Automatic Control in the Department of Electrical Engineering, Chalmers University of Technology, Sweden. From 2005 to 2007, he worked as a senior researcher in control system design at Volvo Technology. For the past decade, he has led a growing group of researchers on battery management systems (BMS). Apart from BMS, his main research interests are in process control and optimal control, with applications motivated by environmental sustainability.

Anton Kersten (kersten@chalmers .se) received his M.Sc. degree in electrical engineering in 2017 from Chalmers University of Technology, Gothenburg, Sweden, where he is currently pursuing his Ph.D. degree. Since 2017, he has been with the Division of Electric Power Engineering, Chalmers University of Technology, Gothenburg, Sweden, where he is involved in the field of multilevel inverters for vehicle powertrains. His research interests include the modeling and control of power electronics for electric drives and the grid integration of renewable energy sources.

Guangzhong Dong (gzdong@ieee .org) received his Ph.D. degree in control science and engineering from the University of Science and Technology of China, Hefei, in 2018. He is a postdoctoral fellow in the Department of Electrical Engineering, Chalmers University of Technology, Gothenburg, Sweden. His research interests include the modeling, estimation, and health prognosis of energy storage systems; optimal energy dispatch; and coordinated control of microgrids.
Changfu Zou (changfu.zou@ chalmers.se) received his Ph.D. degree in automation and control engineering from the University of Melbourne, Australia, in 2017. He is an assistant professor in the Automatic Control Group, Chalmers University of Technology, Gothenburg, Sweden. His research interests include the intelligent management of energy systems and electric vehicles. He serves as an associate editor of IEEE Transactions on Transportation Electrification.

\section{References}

[1] W. Han, C. Zou, L. Zhang, Q. Ouyang, and T. Wik, "Near-fastest battery balancing by cell/ module reconfiguration," IEEE Trans. Smart Grid, vol. 10, no. 6, pp. 6954-6964, Nov. 2019. doi: 10.1109/TSG.2019.2915013.

[2] H. Kim and K. G. Shin, "Scheduling of battery charge, discharge, and rest," in Proc. 30th IEEE Real-Time Syst. Symp., Dec. 2009, pp. 13-22. doi: 10.1109/RTSS.2009.38.

[3] G. Desarnaud, "Second life batteries: A sustainable business opportunity, not a conundrum," Capgemini, Capgemini Invent, Paris, France, Apr. 18, 2019. Accessed: Nov. 26, 2019. [Online]. Available: https://www.capgemini .com/2019/04/second-life-batteries-a-sustainable -business-opportunity-not-a-conundrum/

[4] A. Badam et al., "Software defined batteries," in Proc. ACM 25th Symp. Oper. Syst. Princ., 2015 pp. 215-229. doi: 10.1145/2815400.2815429.

[5] H.-M. Fischer, "Voltage classes for electric mobility," ZVEI- German Electrical and Electronic Manufacturers' Association, Frankfurt, Germany, Dec. 2013. [Online]. Available: https://www.zvei.org/fileadmin/user_upload/ Presse_und_Medien/Publikationen/2014/ april/Voltage_Classes_for_Electric_Mobility/ Voltage_Classes_for_Electric_Mobility.pdf

[6] M. Alahmad, H. Hess, M. Mojarradi, W. West, and J. Whitacre, "Battery switch array system with application for JPL's rechargeable microscale batteries," J. Power Sources, vol. 177 , no. 2 , pp. $566-578,2008$. doi: $10.1016 /$ j.jpowsour.2007.11.053.

[7] S. Ci, J. Zhang, H. Sharif, and M. Alahmad, "A novel design of adaptive reconfigurable multicell battery for power-aware embedded networked sensing systems," in Proc. IEEE Global Telecommun. Conf., Nov. 2007, pp. 1043-1047. doi: 10.1109/GLOCOM.2007.201.

[8] H. Kim and K. G. Shin, "On dynamic reconfiguration of a large-scale battery system," in Proc. 15th IEEE Real-Time Embedded Technol. Appl. Symp., Apr. 2009, pp. 87-96. doi: 10.1109/ RTAS.2009.13.

[9] T. Kim, W. Qiao, and L. Qu, "Power electronicsenabled self-X multicell batteries: A design toward smart batteries," IEEE Trans. Power Electron., vol. 27, no. 11, pp. 4723-4733, Nov. 2012. doi: 10.1109/TPEL.2012.2183618.

[10] B. Lawson, "A software configurable battery," in Proc. 26th Int. Battery, Hybrid Fuel Cell Electr. Veh. Symp., May 2012, pp. 252-263.

[11] Y. Kim et al., "Balanced reconfiguration of storage banks in a hybrid electrical energy storage system," in Proc. IEEE/ACM Int. Conf. Comput.-Aided Design, Nov. 2011, pp. 624-631. doi: 10.1109/ICCAD.2011.6105395.

[12] S. J. Moura, "Estimation and control of battery electrochemistry models: A tutorial," in Proc. 54th IEEE Conf. Decision Control, 2015, pp. 3906-3912. doi: 10.1109/CDC.2015 .7402827 
[13] A. Barré, B. Deguilhem, S. Grolleau, M. Gérard, F. Suard, and D. Riu, "A review on lithium-ion battery ageing mechanisms and estimations for automotive applications," J. Power Sources, vol. 241 , pp. $680-689$, Nov. 2013. doi: 10.1016/j. jpowsour.2013.05.040.

[14] X. Feng, M. Ouyang, X. Liu, L. Lu, Y. Xia, and X. $\mathrm{He}$, "Thermal runaway mechanism of lithium ion battery for electric vehicles: A review," Energy Storage Mater., vol. 10, pp. 246-267, Jan. 2018. doi: 10.1016/j.ensm.2017.05.013.

[15] W. Han, L. Zhang, and Y. Han, "Mathematical modeling, performance analysis and control of battery equalization systems: Review and recent developments," in Advances in Battery Manufacturing, Services, and Management Systems, J. Li, S. Zhou, and Y. Han, Eds. New York: Wiley-IEEE Press, 2016, ch. 12, pp. 281-301.

[16] F. Altaf, L. Johannesson, and B. Egardt, "Simultaneous thermal and state-of-charge balancing of batteries: A review," in Proc. IEEE Veh. Power Propulsion Conf., Oct. 2014, pp. 1-7. doi: 10.1109/VPPC. 2014.7007132.

[17] F. Feng, X. Hu, J. Liu, X. Lin, and B. Liu, "A review of equalization strategies for series battery packs: Variables, objectives, and algorithms," Renew. Sustain. Energy Rev., vol. 116, p. 109,464, Dec. 2019. doi: 10.1016/j.rser.2019. 109464.

[18] Z. Zhang, Y. Cai, Y. Zhang, D. Gu, and Y. Liu, "A distributed architecture based on microbank modules with self-reconfiguration control to improve the energy efficiency in the battery energy storage system," IEEE Trans. Power Electron., vol. 31, no. 1, pp. 304-317, Jan. 2016. doi: 10.1109/TPEL.2015.2406773.

[19] S. K. Mandal, P. S. Bhojwani, S. P. Mohanty, and R. N. Mahapatra, "IntellBatt: Towards smarter battery design," in Proc. 45th ACM/IEEE Design Autom. Conf., June 2008, pp. 872-877. doi: $10.1145 / 1391469.1391690$

[20] J. Andersson, "Lifetime estimation of lithiumion batteries for stationary energy storage systems," M.S. thesis, KTH Royal Inst. of Technol., Stockholm, Sweden, June 2017.

[21] L. C. Casals, B. A. García, and C. Canal, "Second life batteries lifespan: Rest of useful life and environmental analysis," J. Environ. Manage., vol. 232, pp. 354-363, Feb. 2019. doi: 10.1016/j.jenvman.2018.11.046.

[22] B. Molenaar, "Reconfigurable battery system for ultra fast charging of industrial electric vehicles," M.S. thesis, Delft Univ. of Technol., Delft, The Netherlands, May 2010

[23] "Relay contact life," Tyco Electronics Corporation, Winston-Salem, NC, 2009. [Online]. Available: https://www.te.com/commerce/ DocumentDelivery/DDEController?Action=sr chrtrv\&DocNm=13C3236_AppNote\&DocType =CS\&DocLang=EN\#: :text=Contact $\% 20$ life $\% 20$ is $\% 20$ terminated $\% 20$ when, material $\% 20$ loss $\% 20$ due $\% 20$ to $\% 20$ splattering

[24] S. Ci, N. Lin, and D. Wu, "Reconfigurable battery techniques and systems: A survey," IEEE Access, vol. 4, pp. 1175-1189, Mar. 2016. doi: 10.1109/ACCESS.2016.2545338.

[25] L. He et al., "RAC: Reconfiguration-assisted charging in large-scale lithium-ion battery systems," IEEE Trans. Smart Grid, vol. 7, no. 3, pp. 1420-1429, May 2016. doi: 10.1109/ TSG.2015.2450727.

[26] T. Kim, W. Qiao, and L. Qu, "Series-connected self-reconfigurable multicell battery," in Proc. IEEE Appl. Power Electron. Conf. Expo., Mar. 2011, pp. 1382-1387. doi: 10.1109/APEC.2011. 5744772.

[27] T. Kim, W. Qiao, and L. Qu, "A series-connected self-reconfigurable multicell battery capable of safe and effective charging/discharging and balancing operations," in Proc. IEEE Appl. Power Electron. Conf. Expo., Feb. 2012, pp. 2259-2264. doi: 10.1109/APEC.2012.6166137.

[28] M. Momayyezan, B. Hredzak, and V. G. Agelidis, "Integrated reconfigurable converter topology for high-voltage battery systems," IEEE
Trans. Power Electron., vol. 31, no. 3, pp. 19681979, 2016. doi: 10.1109/TPEL.2015.2440441.

[29] T. Morstyn, M. Momayyezan, B. Hredzak, and V. Agelidis, "Distributed control for state-ofcharge balancing between the modules of a reconfigurable battery energy storage system," IEEE Trans. Power Electron., vol. 31, no. 11, pp. 7986-7995, Nov. 2016. doi: 10.1109/ TPEL.2015.2513777.

[30] F. Chen, W. Qiao, and L. Qu, "A modular and reconfigurable battery system," in Proc. IEEE Appl. Power Electron. Conf. Expo., 2017, pp 2131-2135. doi: 10.1109/APEC.2017.7930993.

[31] H. Visairo and P. Kumar, "A reconfigurable battery pack for improving power conversion efficiency in portable devices," in Proc. 7th Int. Caribbean Conf. Devices Circuits Syst., Apr. 2008, pp. 1-6. doi: 10.1109/ICCDCS.2008. 4542628.

[32] P. Kumar, H. Visairo-Cruz, and S. Noble, "Reconfigurable battery pack," U.S. Patent 20090 085553 A1, Apr. 2, 2009.

[33] L. He, L. Gu, L. Kong, Y. Gu, C. Liu, and T. He, "Exploring adaptive reconfiguration to optimize energy efficiency in large-scale battery systems," in Proc. 34th IEEE Real-Time Syst. Symp., Dec. 2013, pp. 118-127. doi: 10.1109/ RTSS.2013.20.

[34] S. Ci, J. Zhang, H. Sharif, and M. Alahmad, "Dynamic reconfigurable multi-cell battery: A novel approach to improve battery performance," in Proc. IEEE Appl. Power Electron. Conf. Expo., 2012, pp. 439-442. doi: 10.1109/ APEC.2012.6165857.

[35] H. Kim and K. G. Shin, "Dynamically reconfigurable framework for a large-scale battery system," U.S. Patent 20100261048 Al, Oct. 14 2010.

[36] H. Kim and K. G. Shin, "DESA: Dependable, efficient, scalable architecture for managemen of large-scale batteries," IEEE Trans. Ind. Informat., vol. 8, no. 2, pp. 406-417, May 2012. doi: 10.1109/TII.2011.2166771

[37] S. Steinhorst et al., "Distributed reconfigurable battery system management architectures," in Proc. 21st Asia South Pacific Design Autom. Conf., Jan. 2016, pp. 429-434. doi: 10.1109/ASPDAC. 2016.7428049.

[38] L. He, E. Kim, and K. G. Shin, "A case study on improving capacity delivery of battery packs via reconfiguration," ACM Trans. Cyber-Phys. Syst., vol. 1, no. 2, pp. 1-23, 2017. doi: 10.1145 3035539.

[39] V. Sukumar et al., "Switch array system for thin film lithium microbatteries," J. Power Sources, vol. 136, no. 2, pp. 401-407, Oct. 2004 doi: 10.1016/j.jpowsour.2004.03.033.

[40] J. Cabrera, A. Vega, F. Tobajas, V. Déniz, and H. A. Fabelo, "Design of a reconfigurable Li-ion battery management system (BMS)," in Proc. XI Tecnologias Aplicadas a la Ensenanza de la Electronica (Technol. Appl. Electron. Teaching), June 2014, pp. 1-6. doi: 10.1109/TAEE.2014.6900162.

[41] G. Wang, J. Pou, and V. G. Agelidis, "Reconfigurable battery energy storage system for utilityscale applications," in Proc. 41st Annu. Conf. IEEE Ind. Electron. Soc., Nov. 2015, pp. $004086-$ 004091. doi: 10.1109/IECON.2015.7392737.

[42] N. Bouchhima, M. Schnierle, S. Schulte, and K. P. Birke, "Active model-based balancing strategy for self-reconfigurable batteries," J. Power Sources, vol. 322, pp. 129-137, Aug. 2016. doi: 10.1016/j.jpowsour.2016.05.027.

[43] L. He, Z. Yang, Y. Gu, C. Liu, T. He, and K. G. Shin, "SoH-Aware reconfiguration in battery packs," IEEE Trans. Smart Grid, vol. 9, no. 4, pp. 3727-3735, July 2018. doi: 10.1109/TSG.2016. 2639445.

[44] N. Lin and S. Ci, "Toward dynamic programming-based management in reconfigurable battery packs," in Proc. IEEE Appl. Power Elec tron. Conf. Expo., Mar. 2017, pp. 2136-2140. doi: 10.1109/APEC.2017.7930994.

[45] W. Han and L. Zhang, "Battery cell reconfiguration to expedite charge equalization in series- connected battery systems," IEEE Robot. Autom. Lett., vol. 3, no. 1, pp. 22-28, Jan. 2018. doi 10.1109/LRA.2017.2728204.

[46] S. Abhinav, G. Binetti, A. Davoudi, and F. L. Lewis, "Toward consensus-based balancing of smart batteries," in Proc. IEEE Appl. Power Electron. Conf. Expo., Mar. 2014, pp. 2867-2873. doi: 10.1109/APEC.2014.6803711.

[47] M. Doyle, T. F. Fuller, and J. Newman, "Modeling of galvanostatic charge and discharge of the lithium/polymer/insertion cell," $J$. Electrochemical Soc., vol. 140, no. 6, pp. 1526-1533, 1993. doi: 10.1149/1.2221597.

[48] C. Zou, C. Manzie, and D. Nesić, "A framework for simplification of PDE-based lithium-ion battery models," IEEE Trans. Control Syst. Technol., vol. 24, no. 5, pp. 1594-1609, Sept. 2016. doi: 10.1109/TCST.2015.2502899.

[49] Y. Li, M. Vilathgamuwa, T. Farrell, S. S. Choi, N. T. Tran, and J. Teague, "A physics-based distributed-parameter equivalent circuit mode for lithium-ion batteries," Electrochim. Acta, vol. 299, pp. 451-469, Mar. 2019. doi: 10.1016/j. electacta.2018.12.167.

[50] E. Chemali, P. J. Kollmeyer, M. Preindl, R. Ahmed, and A. Emadi, "Long short-term memory networks for accurate state-of-charge estimation of Li-ion batteries," IEEE Trans. Ind. Electron., vol. 65, no. 8, pp. 6730-6739, Aug. 2018. doi: 10.1109/TIE.2017.2787586.

[51] X. Hu, H. Yuan, C. Zou, Z. Li, and L. Zhang, "Co-estimation of state of charge and state of health for lithium-ion batteries based on fractional-order calculus," IEEE Trans. Veh. Technol., vol. 67, no. 11, pp. 10,319-10,329, Nov. 2018. doi: 10.1109/TVT.2018.2865664.

[52] X. Tang, C. Zou, K. Yao, J. Lu, Y. Xia, and F. Gao, "Aging trajectory prediction for lithium-ion batteries via model migration and Bayesian Monte Carlo method," Appl. Energy, vol. 254, p. 113,591, Nov. 2019. doi: 10.1016/j.apenergy.2019. 113591

[53] X. Hu, S. Li, and H. Peng, "A comparative study of equivalent circuit models for Li-ion batteries," J. Power Sources, vol. 198, pp. 359-367, Jan. 2012. doi: 10.1016/j.jpowsour.2011.10.013.

[54] B. J. Yurkovich, "Electrothermal battery pack modeling and simulation," M.S. thesis, The Ohio State Univ., Columbus, 2010.

[55] W. Han, C. Zou, C. Zhou, and L. Zhang, "Estimation of cell SOC evolution and system performance in module-based battery charge equalization systems," IEEE Trans. Smart Grid, vol. 10, no. 5, pp. 4717-4728, Sept. 2019. doi: 10.1109/TSG.2018.2867017.

[56] J. Fu, "Fundamentals of on-resistance in load switches," Texas Instruments, Dallas, June 2016. [Online]. Available: https://www.ti.com/ lit/an/slva771/slva771.pdf?ts=1602833244742\&ref_ url=https $\% 253 \mathrm{~A} \% 252 \mathrm{~F} \% 252 \mathrm{Fwww}$.google. com $\% 252 \mathrm{~F} \#: \sim:$ text=The $\% 20$ combined $\% 20$ effect $\% 20$ of $\% 20$ all,when $\% 20$ selecting $\% 20 \mathrm{a} \% 20$ load $\% 20$ switch.\&text=As $\% 20$ power $\% 20$ is $\% 20$ dissipated $\% 20$ through, and $\% 20$ lifetime $\% 20$ of $\% 20$ the $\% 20$ device.

[57] A. Sattar, "Power MOSFET basics," IXYS Corporation, Milpitas, CA, 2007.

[58] "AN-9010 MOSFET basics," Fairchild Semiconductor, Sunnyvale, CA, 2013. [Online]. Available: https://www.onsemi.cn/pub/Collateral/ AN-9010.pdf.pdf

[59] N. Lin, S. Ci, D. Wu, and H. Guo, "An optimization framework for dynamically reconfigurable battery systems," IEEE Trans. Energy Convers., vol. 33, no. 4, pp. 1669-1676, Dec. 2018. doi: 10.1109/TEC.2018.2850853.

[60] D. S. Naidu, Optimal Control Systems. Boca Raton, FL: CRC Press, 2002.

[61] F. Jin and K. G. Shin, "Pack sizing and reconfiguration for management of large-scale batteries," in Proc. IEEE/ACM Int. Conf. Cyber-Phys. Syst., Apr. 2012, pp. 138-147. doi: 10.1109/ICCPS.2012.22 